\title{
P35
}

\section{APPLICABILITY OF THE PREDICTIVE FORMULAE FOR SUPENDED SEDIMENT CONCENTRATION ON FULL-SCALE RIPPLED BED AND SHEET FLOW}

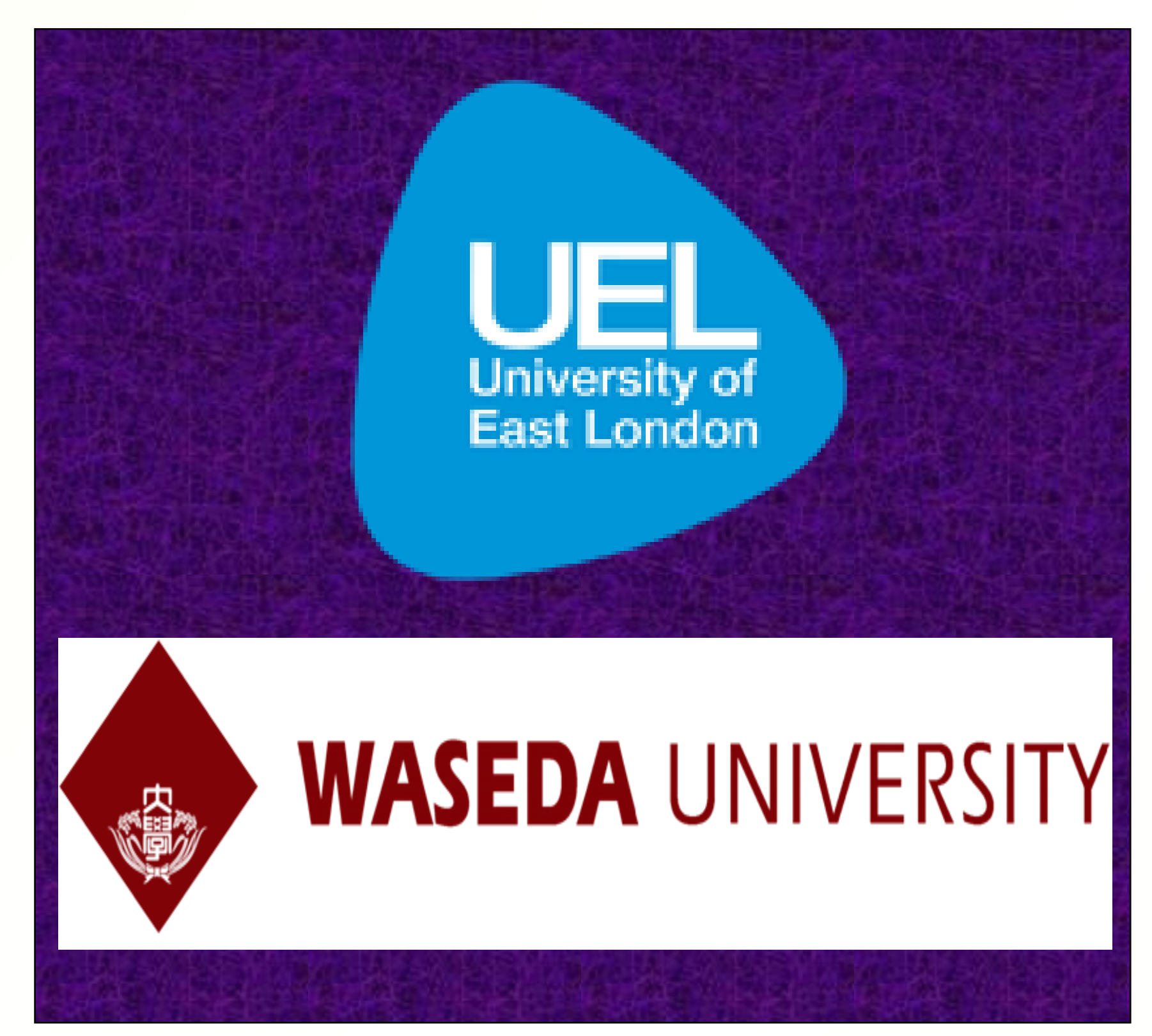

\section{Ravindra Jayaratne ${ }^{1}$ \& Tomoya Shibayama² \\ E-mail: ${ }^{1}$ r.jayaratne@uel.ac.uk, ${ }^{2}$ shibayama@waseda.jp}

\section{INTRODUCTION}

Purpose: To investigate the applicability and performance of Suspended Sediment Concentration Models of Jayaratne \& Shibayama (2007) using fullscale rippled bed and sheet flow laboratory data in SANTOSS database.

Methodology: Dimensional Analysis \& Best-Fit Technique (Regression analysis) are the main methods used to revise the previous sets of theoretical models of Jayaratne \& Shibayama (2007).

$\square$ Innovative Points: Two suspension layers (Lower \& Upper) were identified over rippled beds, therefore two separate theoretical models were proposed whereas four suspension layers (Pick-up layer, Upper sheet flow layer, Transition layer \& Suspension layer) were identified over sheet flow and models were proposed for upper sheet flow and suspension layers due to the availability of measured data.

\section{REVISED MODELS}

\begin{tabular}{|l|c|c|}
\hline \multicolumn{3}{|c|}{ FULL SCALE RIPPLED-BED (75 DATA SETS) } \\
\hline & Lower Suspension Layer & Upper Suspension Layer \\
\hline $\begin{array}{l}\text { Reference } \\
\text { concentration }\end{array}$ & \multicolumn{2}{|c|}{$c_{r}=\frac{k_{1} \psi v}{\sqrt{(s-1) g d}(\eta / 2)}$} \\
\hline $\begin{array}{l}\text { Diffusion } \\
\text { coefficient }\end{array}$ & $\varepsilon_{r}=k_{2} u_{w_{w c}} A_{t_{r}}\left(\frac{w_{s}}{u_{w_{w c}}}\right)^{2}\left(\frac{\eta}{d}\right)^{0.1}\left(\frac{\lambda}{d}\right)^{0.25} d_{*}^{-1.5}$ & $M_{r}=k_{3}\left(\frac{\eta}{d}\right)^{0.1}\left(\frac{\lambda}{d}\right)^{0.25} d_{s}^{-1.5}$ \\
\hline $\begin{array}{l}\text { Sediment } \\
\text { concentration } \\
\text { profile }\end{array}$ & $c(z)=c_{r} \exp \left\{\frac{-w_{s}(z-r)}{\varepsilon_{r}}\right\}$ & $c(z)=c_{r}\left(\frac{z_{0}}{z}\right)^{M_{r}}$ \\
\hline
\end{tabular}

\begin{tabular}{|c|c|c|}
\hline \multicolumn{3}{|c|}{ FULL SCALE SHEET-FLOW (8O DATA SETS) } \\
\hline & Upper Sheet-Flow Layer & Suspension Layer \\
\hline $\begin{array}{l}\text { Reference } \\
\text { concentration }\end{array}$ & \multicolumn{2}{|c|}{$c_{s}=\frac{k_{4} \theta_{V}}{\sqrt{(s-1) g d} d}$} \\
\hline $\begin{array}{l}\text { Diffusion } \\
\text { coefficient }\end{array}$ & \multicolumn{2}{|c|}{$M_{s}=\left(\frac{w_{s}}{U_{w w c}}\right)^{k_{s}}$} \\
\hline $\begin{array}{l}\text { Sediment } \\
\text { concentration } \\
\text { profile }\end{array}$ & $c(z)=c_{s} \exp \left\{\frac{-\left(z-k_{s} d\right)}{5 M_{s} d}\right\}$ & $c(z)=c_{s}\left(\frac{25 d}{z}\right)^{M_{s}}$ \\
\hline
\end{tabular}

\section{JAYARATNE \& SHIBAYAMA (2007) PREDICTIVE MODELS}

A set of predictive models were formulated for computing suspended sediment concentration in and outside the surf zone under three suspension mechanisms:
(a) Turbulent motion over sand ripples,
(b) Sheet flow layer, and
(c) Turbulent motion under breaking waves.

$\square$ Reference concentration and diffusion coefficient were formulated from Dimensional analysis and Best-fit technique.

Model parameters were calibrated with the help of published laboratory and field data.

Time-averaged concentration profiles were derived from the steady diffusion equation

- Published experimental data from 19 sources (325 cases) from 1977 to 1996 were better explained by the proposed formulae.
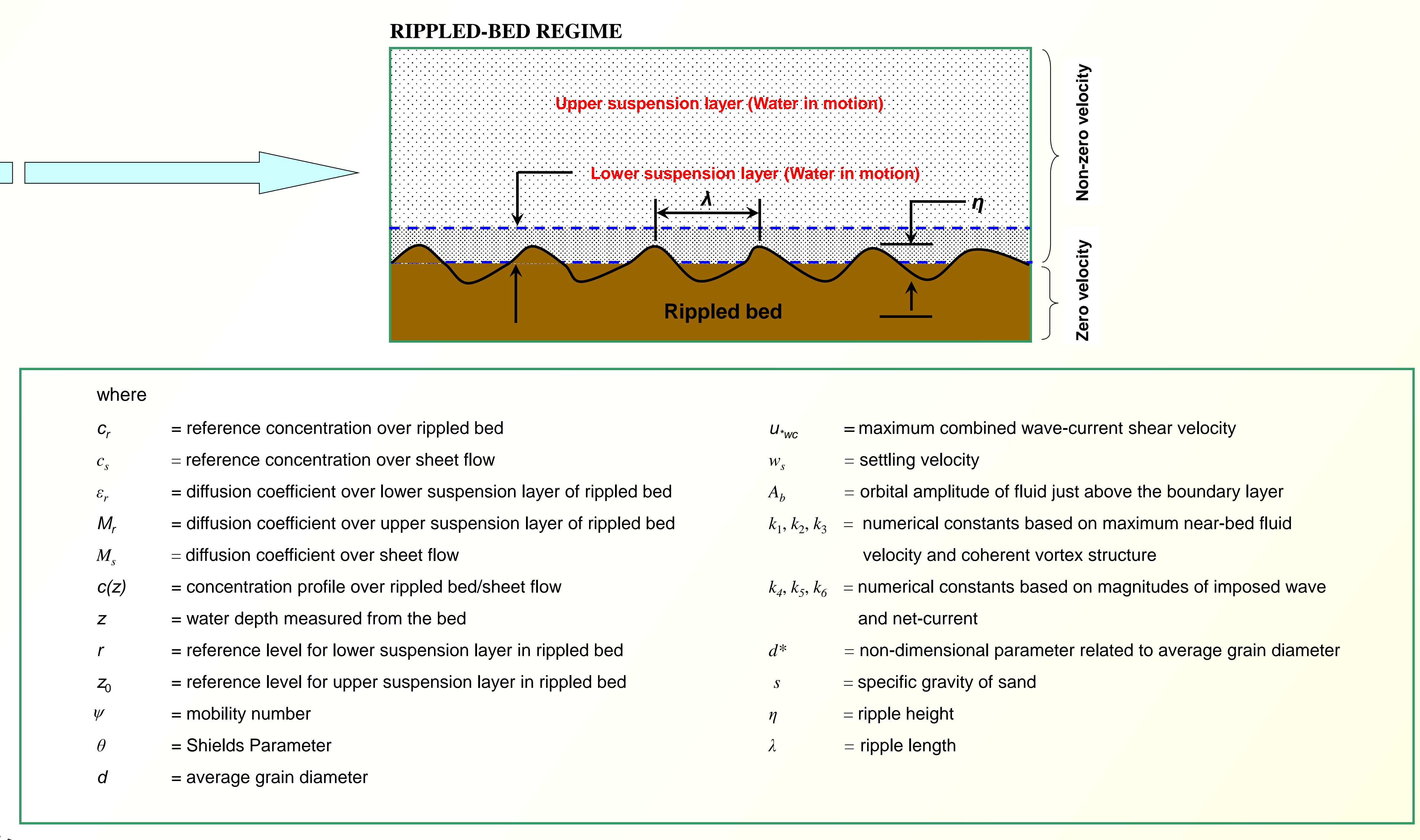

From the measured experimental data of Katopodi et al. (1994)

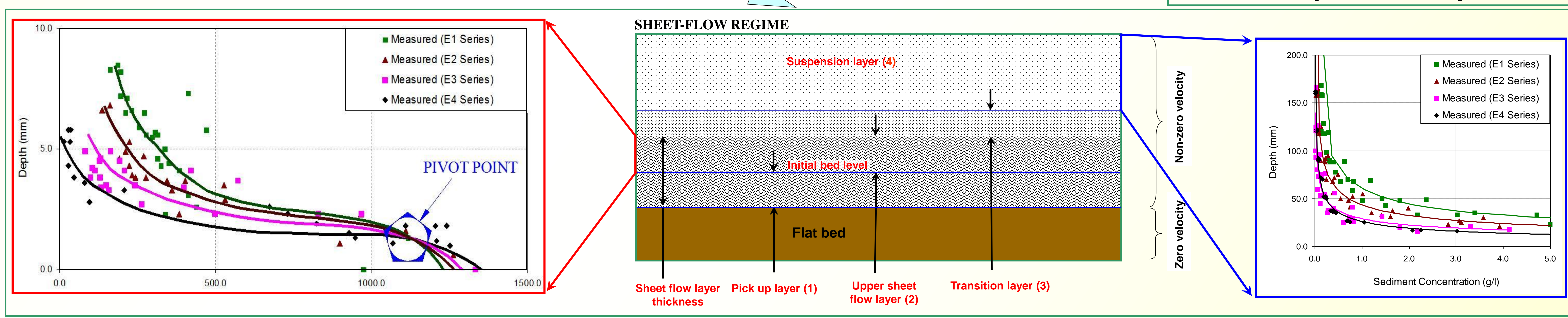

4. MODEL RESULTS

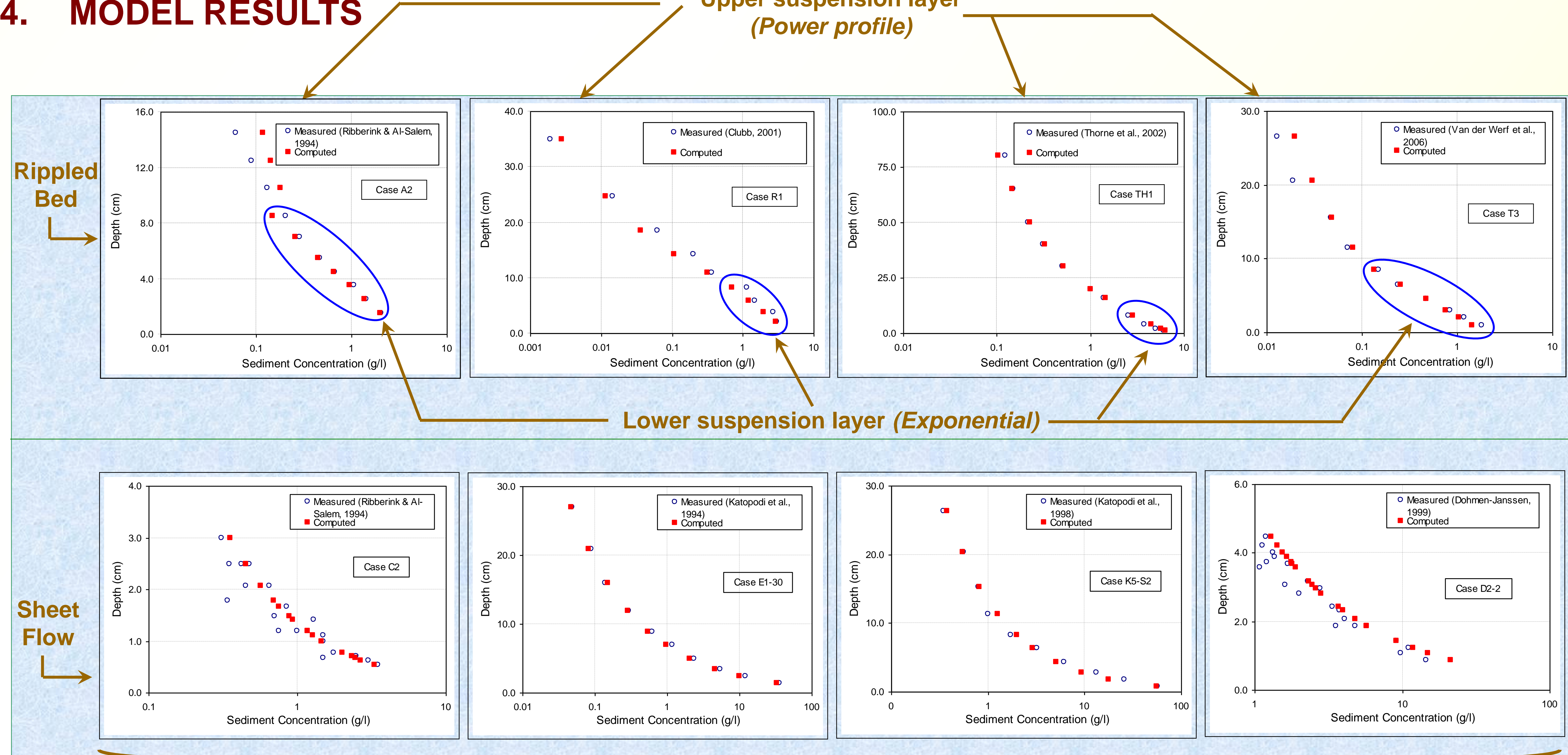

Suspension layer (Power profile)
5. DISCUSSION

$\square$ In rippled bed regime, time-averaged concentration profile over lower suspension layer shows an exponential form of behaviour with depth whereas it is over upper suspension layer shows a power relationship. The numerical coefficients $\left(k_{1}, k_{2}, k_{3}\right)$ in revised predicted formulae depends on the maximum near-bed fluid velocity and coherent vortex structure.

口 Similarly, in sheet flow regime the time-averaged concentration profile over upper sheet flow layer shows an exponential form of behaviour with depth, and conversely it is over suspension layer shows a power relationship. The numerical coefficients $\left(k_{4}, k_{5}\right.$, $k_{6}$ ) in revised predicted formulae depends on the magnitudes of imposed wave and net-current.

\section{CONCLUSIONS}

There is a close match between sediment concentration predicted by the revised rippled bed and sheet flow models and the measured data.

From the measured data of Katopodi et al. (1994), in sheet flow regime, when the wave period remains constant, different combination of wave and net-current conditions acting on a particular sand form a Single Pivot Point on the curves of measured time-averaged concentration profiles. 